Orthopäde $2017 \cdot 46: 111-113$

DOI 10.1007/s00132-016-3374-2

C) Springer Medizin Verlag Berlin 2017

CrossMark

\section{B. M. Holzapfel · M. Rudert}

Orthopädische Klinik König-Ludwig Haus, Julius-Maximilians Universität Würzburg, Würzburg, Deutschland

\title{
Pfannenrevision in der Alloarthroplastik des Hüftgelenks
}

\section{Aktuelle Therapiekonzepte und neue Entwicklungen}

Sehr geehrte Leserinnen und Leser,

Daten aus Deutschland, den USA und dem United Kingdom zeigen eine deutliche Zunahme der Prävalenz von Hüftprothesenimplantationen in den letzten Jahrzehnten [1-3]. Während einige Studien eine weitere Zunahme - auch der Prävalenz von Revisionseingriffen - erwarten [4-6], lassen bevölkerungsbezogene Analysen aus Deutschland vermuten, dass zumindest bei Patienten mit einem Alter von über 70 Jahren ein Plateau erreicht scheint $[7,8]$. Unabhängig von den Einschätzungen der allgemeinen Versorgungssituation konnte gezeigt werden, dass gerade Kliniken mit hohem Spezialisierungsgrad mit einer weiter steigenden Anzahl an Revisionseingriffen rechnen müssen [9]. So kam es beispielsweise in unserer Klinik zwischen 2005 und $2015 \mathrm{zu}$ einem Anstieg der durchgeführten Kniegelenks- und Hüftgelenksprothesenwechsel um $306 \%$ bzw. $205 \%$.

》) Moderne Revisionssysteme machen eine intraoperative Anpassung des Implantats an den ossären Defekt möglich

Um eine hohe Versorgungsqualität durch Spezialisierung gewährleisten zu können, ist eine gezielte Aus- und Weiterbildung essenziell. Hierzu bedarf es einer regelmäßigen Aufarbeitung der bestehenden Literatur und Überarbeitung eigener Standards. Mit der Zusammenstellung dieses Heftes haben wir versucht, Ihnen diese Arbeit zu erleichtern. Die Darstellung aktueller Therapiestrategien beim Pfannenwechsel aus Sicht verschiedener hoch spezialisierter Zentren bildet dabei den Kern des Heftes.

Für den unkomplizierten Pfannenwechsel stehen dem behandelnden Chirurgen viele verschiedene Implantatsysteme zur Verfügung, und es können mittlerweile reproduzierbar gute klinische Ergebnisse erzielt werden. Bei Vorliegen großer periprothetischer Knochendefekte oder nach Mehrfachwechseln ist die Datenlage nicht klar und es fehlen weitgehend Handlungsempfehlungen, die auf Studien mit hohem Evidenzlevel beruhen. In diesen Fällen ist ein differenziertes Vorgehen notwendig, mit exakter präoperativer Planung und Abschätzung der Defektausmaße. Die intraoperative Realität ist hierbei jedoch auch durch den Einsatz moderner bildgebender Verfahren nicht immer sicher vorherzusagen. Deshalb ist der Einsatz eines defektadaptierten Therapieprotokolls zu empfehlen, das durch Anpassung des Implantatsystems bzw. der Versorgungsstrategie an den Defekt eine gewisse intraoperative Flexibilität erlaubt. Ein weiterer Vorteil eines defektadaptierten Behandlungsplanes ist die Möglichkeit eines Vergleiches klinischer Ergebnisse unterschiedlicher Studien, abhängig vom vorliegenden Defekt und verwendeten Implantattyp. In Zukunft mag dies die Auswahl eines passgenauen Implantates bzw. einer geeigneten rekonstruktiven $\mathrm{Maßnahme} \mathrm{erleichtern} \mathrm{und} \mathrm{letztendlich}$ zur Verbesserung klinischer Ergebnisse nach Pfannenwechsel führen.

Wir freuen uns sehr über die Beiträge der einzelnen Autorengruppen und möchten uns an dieser Stelle für die mühevolle Erstellung der Manuskripte bedanken. Wir wünschen Ihnen viel Freude beim Lesen und hoffen, dass die vermittelten Informationen Ihren klinischen Alltag bereichern.

Mit besten kollegialen Grüßen,

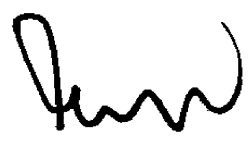

Boris M. Holzapfel und

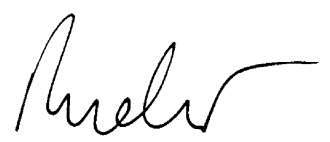

Maximilian Rudert.

\section{Korrespondenzadresse}

Dr. med. B. M. Holzapfel, Ph.D., FEBOT

Orthopädische Klinik König-Ludwig Haus, Julius-Maximilians Universität Würzburg Brettreichstr. 11, 97074 Würzburg, Deutschland b-holzapfel.klh@uni-wuerzburg.de

\section{Prof. Dr. med. M. Rudert}

Orthopädische Klinik König-Ludwig Haus, Julius-Maximilians Universität Würzburg Brettreichstr. 11, 97074 Würzburg, Deutschland m-rudert.klh@uni-wuerzburg.de

Interessenkonflikt. B. M. Holzapfel und M. Rudert geben an, dass kein Interessenkonflikt besteht. 


\section{Literatur}

1. Statistisches Bundesamt (2015) Bevölkerung Deutschlands bis 2060; 13 . koordinierte Bevölkerungsvorausberechnung. https://www. destatis.de/DE/Publikationen/Thematisch/ Bevoelkerung/VorausberechnungBevoelkerung/ BevoelkerungDeutschland2060Presse 512420 4159004.pdf?_blob=publicationFile. Zugegriffen: 23.06.2016

2. Maradit Kremers $H$, Larson DR, Crowson CS, Kremers WK, Washington RE, Steiner CA, Jiranek WA, Berry DJ (2015) Prevalence of Total Hip and Knee Replacement in the United States. J Bone Joint Surg Am 97(17):1386-1397

3. Wengler A, Nimptsch U, Mansky T (2014) Hip and knee replacement in Germany and the USA: Analysis of individual inpatient data from German and US hospitals for the years 2005 to 2011. Dtsch Ärztebl Int 111(23-24):407-416

4. Culliford D, Maskell J, Judge A, Cooper C, PrietoAlhambra D, Arden NK, Group COS (2015) Future projections of total hip and knee arthroplasty in the UK: results from the UK Clinical Practice Research Datalink. Osteoarthritis Cartilage 23(4):594-600

5. Kurtz S, Ong K, Lau E, Mowat F, Halpern M (2007) Projections of primary and revision hip and knee arthroplasty in the United States from 2005 to 2030. JBone Joint Surg Am 89(4):780-785

6. Patel A, Pavlou G, Mujica-Mota RE, Toms AD (2015) The epidemiology of revision total knee and hip arthroplasty in England and Wales: a comparative analysis with projections for the United States. A study using the National Joint Registry dataset. Bone Joint J 97-B(8):1076-1081

7. Institut für angewandte Qualitätsförderung und Forschung im Gesundheitswesen $\mathrm{GmbH}$ (2014) AQUA. Qualitätsreport 2013. http://www.sqg.de/ sqg/upload/CONTENT/Qualitaetsberichte/2013/ AQUA-Qualitaetsreport-2013.pdf. Zugegriffen: 23.06.2016

8. Statistisches Bundesamt (2014) Fallpauschalenbezogene Krankenhausstatistik (DRGStatistik) Operationen und Prozeduren der vollstationären Patientinnen und Patienten in Krankenhäusern - Ausführliche Darstellung 2013. https://www.destatis.de/DE/Publikationen/ Thematisch/Gesundheit/Krankenhaeuser/ OperationenProzeduren5231401137014.pdf? blob=publicationFile. Zugegriffen:23.06.2016

9. Kurtz SM, Ong KL, Schmier J, Zhao K, Mowat F, Lau E (2009) Primary and revision arthroplasty surgery caseloads in the United States from 1990 to 2004. J Arthroplasty 24(2):195-203
Fachnachrichten
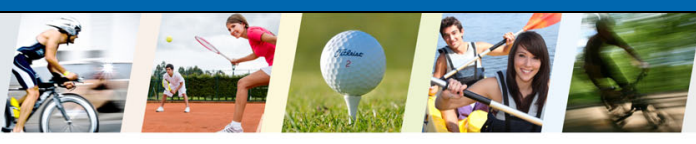

REGENSBURGER SPORTTAGE

30. 5. - 2.6. 2017

\section{Regensburger Sporttage 2017}

Viertageskurs Sportmedizin nach dem neuem Curriculum vom 30.05.-02.06.2017

Die 17. Regensburger Sporttage bieten ein abwechslungsreiches sportmedizinisches Programm mit Top-Referenten und attraktivem Sportpraxis-Teil.

Zum siebzehnten Mal veranstaltet die Orthopädische Universitätsklinik Regensburg am Asklepios Klinikum Bad Abbach in diesem Jahr vom 30.05.-02.06.2017 die "Regensburger Sporttage" für Ärzte. Sportmediziner, Sportwissenschaftler und Sportphysiotherapeuten mit langjähriger Erfahrung im hochklassigen Amateuer- und Spitzensport geben dabei detaillierte Hintergrundinfomationen und konkrete Handlungsempfehlungen für ein optimales Therapieergebnis in der medizinischen Betreuung von Sportlern. Ausgewählte Referenten aus der Fußball- und Basketballbundesliga vermitteln in diesem Jahr besondere Einblicke in moderne Behandlungskonzepte bei Verletzungen am Bewegungssystem. Mit dem Sono-Workshop und einer Einführung ins Medical Taping finden darüber hinaus wichtige sportmedizinische Grundlagentechniken Platz im Programm.

Technische Hintergründe und Spaß an der Bewegung vermitteln die hochklassigen sportwissenschaftlichen Instruktoren der Universität Regensburg in den diesjährigen Schwerpunktsportarten Tennis, Radsport und Golf. Eine spannende Kanutour auf der Donau durch den "Donaudurchbruch" bei Weltenburg rundet das Sportprogramm erlebnisreich ab. Für die Regensburger Sporttage bieten der malerisch gelegene Ort Bad Abbach und die Weltkulturerbestadt Regensburg eine hervorragende Kulisse.

\section{Wissenschaftliche Leitung}

- Prof. Dr. med. Dr. h. c. J. Grifka

- Prof. Dr. med. T. Renkawitz

- PD Dr. med. C. Baier

\section{Anerkennung}

Der Kurs erfüllt die Anforderungen für die Zweitageskurse 4 und 9 nach dem neuen Curriculum der DGSP zur Erlangung der Zusatzweiterbildung „Sportmedizin“ mit 16 Stunden Theorie und Praxis der Sportmedizin und 16 Stunden sportmedizinische Aspekte des Sports.

Die Fort- und Weiterbildungsveranstaltung wird bei der BLÄK beantragt.

Physiotherapeuten erhalten für jeden Tag der Fortbildungsveranstaltung eine Teilnahmebestätigung über 8 Unterrichtseinheiten entsprechend 8 Fortbildungspunkten.

\section{Anmeldung und Information}

Orthopädische Universitätsklinik Regensburg

Asklepios Klinikum Bad Abbach GmbH

93077 Bad Abbach

Telefax: 09405.182479

E-Mail: erika.blumenstock@klinik.uni-regensburg.de

www.uni-r.de/orthopaedie/sporttage

\section{Bildnachweis}

Jamie King@flickr.com | Mariano Kamp@flickr.com | auremar@fotolia.com |

Andres Rodriguez@fotolia.com | Tord Sollie@flickr.com 
Hier steht eine Anzeige.

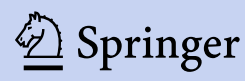

